\title{
Multiple solutions to weakly coupled supercritical elliptic systems
}

\author{
Omar Cabrera ${ }^{1} \cdot$ Mónica Clapp ${ }^{1}$
}

Received: 30 August 2018 / Accepted: 18 December 2018 / Published online: 2 January 2019 (c) Fondazione Annali di Matematica Pura ed Applicata and Springer-Verlag GmbH Germany, part of Springer Nature 2019

\section{Abstract}

We study a weakly coupled supercritical elliptic system of the form

$$
\begin{cases}-\Delta u=\left|x_{2}\right|^{\gamma}\left(\mu_{1}|u|^{p-2} u+\lambda \alpha|u|^{\alpha-2}|v|^{\beta} u\right) & \text { in } \Omega, \\ -\Delta v=\left|x_{2}\right|^{\gamma}\left(\mu_{2}|v|^{p-2} v+\lambda \beta|u|^{\alpha}|v|^{\beta-2} v\right) & \text { in } \Omega, \\ u=v=0 & \text { on } \partial \Omega,\end{cases}
$$

where $\Omega$ is a bounded smooth domain in $\mathbb{R}^{N}, N \geq 3, \gamma \geq 0, \mu_{1}, \mu_{2}>0, \lambda \in \mathbb{R}, \alpha, \beta>1$, $\alpha+\beta=p$, and $p \geq 2^{*}:=\frac{2 N}{N-2}$. We assume that $\Omega$ is invariant under the action of a group $G$ of linear isometries, $\mathbb{R}^{N}$ is the sum $F \oplus F^{\perp}$ of $G$-invariant linear subspaces, and $x_{2}$ is the projection onto $F^{\perp}$ of the point $x \in \Omega$. Then, under some assumptions on $\Omega$ and $F$, we establish the existence of infinitely many fully nontrivial $G$-invariant solutions to this system for $p \geq 2^{*}$ up to some value which depends on the symmetries and on $\gamma$. Our results apply, in particular, to the system with pure power nonlinearity $(\gamma=0)$ and yield new existence and multiplicity results for the supercritical Hénon-type equation

$$
-\Delta w=\left|x_{2}\right|^{\gamma}|w|^{p-2} w \quad \text { in } \Omega, \quad w=0 \quad \text { on } \partial \Omega .
$$

Keywords Weakly coupled elliptic system · Bounded domain - Supercritical nonlinearity · Hénon-type equation · Phase separation

Mathematics Subject Classification 35J47 $\cdot 35 \mathrm{~B} 33 \cdot 35 \mathrm{~B} 40 \cdot 35 \mathrm{~J} 50$

M. Clapp was partially supported by UNAM-DGAPA-PAPIIT Grant IN100718 (Mexico).

$凶$ Mónica Clapp

monica.clapp@im.unam.mx

Omar Cabrera

omar.cabrera@im.unam.mx

1 Instituto de Matemáticas, Universidad Nacional Autónoma de México, Circuito Exterior, Ciudad Universitaria, 04510 Coyoacán, CDMX, Mexico 


\section{Introduction}

We consider the weakly coupled elliptic system

$$
\begin{cases}-\Delta u=\left|x_{2}\right|^{\gamma}\left(\mu_{1}|u|^{p-2} u+\lambda \alpha|u|^{\alpha-2}|v|^{\beta} u\right) & \text { in } \Omega, \\ -\Delta v=\left|x_{2}\right|^{\gamma}\left(\mu_{2}|v|^{p-2} v+\lambda \beta|u|^{\alpha}|v|^{\beta-2} v\right) & \text { in } \Omega, \\ u=v=0 & \text { on } \partial \Omega,\end{cases}
$$

where $\Omega$ is a bounded smooth domain in $\mathbb{R}^{N}, N \geq 3, \gamma \geq 0, \mu_{1}, \mu_{2}>0, \lambda \in \mathbb{R}, \alpha, \beta>1$, $\alpha+\beta=p$, and $p \in(2, \infty)$. The space $\mathbb{R}^{N}$ is decomposed into a direct sum $\mathbb{R}^{N}=F \oplus F^{\perp}$, where $F^{\perp}$ is the orthogonal complement of $F$, and $x_{2}$ is the orthogonal projection onto $F^{\perp}$ of the point $x \in \Omega$.

Systems of this type arise as a model for various physical phenomena. In particular, the cubic system, where $N=3, p=4, \alpha=\beta$ and $\gamma=0$, appears in nonlinear optics and in the study of standing waves in a double mixture of Bose-Einstein condensates, and has received much attention in recent years. There is an extensive literature on subcritical systems with $p<2_{N}^{*}:=\frac{2 N}{N-2}$ and $\gamma=0$. We refer to [25] for a detailed account.

When $\gamma=0$ and $p$ is the critical Sobolev exponent $2_{N}^{*}$, existence and multiplicity results, both in bounded domains and in $\mathbb{R}^{N}$, were recently obtained in $[6,9,15,21,22]$. Critical systems of Brezis-Nirenberg type have been studied in [4,5,18,23].

Here we shall, mainly, focus our attention on the supercritical case $p>2_{N}^{*}$.

When $\lambda=0$ the system (1.1) reduces to the problem

$$
\begin{cases}-\Delta w=\left|x_{2}\right|^{\gamma}|w|^{p-2} w & \text { in } \Omega, \\ w=0 & \text { on } \partial \Omega .\end{cases}
$$

Note that if $w$ solves $(1.2)$, then $\left(\mu_{1}^{1 /(2-p)} w, 0\right)$ and $\left(0, \mu_{2}^{1 /(2-p)} w\right)$ solve the system $(1.1)$ for every $\lambda$. Solutions of this type are called semitrivial. We are interested in fully nontrivial solutions to (1.1), i.e., solutions where both components, $u$ and $v$, are nontrivial. A solution is said to be synchronized if it is of the form ( $s w, t w)$ with $s, t \in \mathbb{R}$, and it is called positive if $u \geq 0$ and $v \geq 0$. The system (1.1) is called cooperative if $\lambda>0$ and competitive if $\lambda<0$.

In the cooperative case, we make the following additional assumption:

(A) If $\lambda>0$, then there exists $r \in(0, \infty)$ such that

$$
\mu_{1} r^{p-2}+\lambda \alpha r^{\alpha-2}-\lambda \beta r^{\alpha}-\mu_{2}=0 .
$$

We consider symmetric domains. Our setting is as follows.

Let $G$ be a closed subgroup of the group $O(N)$ of linear isometries of $\mathbb{R}^{N}$. We write $G x:=\{g x: g \in G\}$ for the $G$-orbit of a point $x \in \mathbb{R}^{N}$. Recall that a subset $X$ of $\mathbb{R}^{N}$ is called $G$-invariant if $G x \subset X$ for every $x \in X$ and a function $u: X \rightarrow \mathbb{R}$ is $G$-invariant if $u$ is constant on $G x$ for every $x \in X$.

We assume that the domain $\Omega$ and the linear subspace $F$ of $\mathbb{R}^{N}$ are $G$-invariant and satisfy

$\left(F_{1}\right) \quad F \neq \mathbb{R}^{N}$ if $\gamma>0$,

$\left(F_{2}\right) \Omega_{0}:=\{x \in \Omega: \operatorname{dim} G x=0\} \subset F$.

We are interested in finding $G$-invariant solutions $(u, v)$ to the system (1.1), i.e., both components $u$ and $v$ are $G$-invariant. We denote by

$$
d:=\min \left\{\operatorname{dim} G x: x \in \Omega \backslash \Omega_{0}\right\}>0,
$$


and, for $p \in[1, \infty)$, we set

$$
\gamma_{p}:=p\left(\frac{N}{2}-\frac{N}{p}-1\right) .
$$

We write $2_{k}^{*}$ for the critical Sobolev exponent in dimension $k$, i.e., $2_{k}^{*}:=\frac{2 k}{k-2}$ if $k>2$ and $2_{k}^{*}:=\infty$ if $k \leq 2$. Note that $\gamma_{p} \geq 0$ if $p \geq 2_{N}^{*}$. We will prove the following result.

Theorem 1.1 Assume $(A),\left(F_{1}\right)$ and $\left(F_{2}\right)$, and let $p \in\left(2,2_{N-d}^{*}\right)$. If $\Omega_{0} \neq \emptyset$ we assume further that $\gamma>\max \left\{\gamma_{p}, 0\right\}$. Then, the system (1.1) has infinitely many fully nontrivial $G$-invariant solutions, one of which is positive.

Note that as $d>0$, we have that $2_{N-d}^{*}>2_{N}^{*}$. For $\lambda>0$, the solutions given by Theorem 1.1 are synchronized and infinitely many of them are sign-changing. In contrast, as shown in [9, Proposition 2.3], there are no synchronized solutions for $\lambda$ smaller than some number $\lambda_{*}<0$. Moreover, the positive solution given by Theorem 1.1 has minimal energy among all fully nontrivial $G$-invariant solutions when $\lambda<0$.

We state some special cases of Theorem 1.1. Firstly, if $\Omega_{0}=\emptyset$, we may take $\gamma=0$, and our result reads as follows.

Corollary 1.2 Assume (A). If $\operatorname{dim} G x \geq d>0$ for every $x \in \Omega$, then, for any $p \in$ $\left[2_{N}^{*}, 2_{N-d}^{*}\right)$, the system

$$
\begin{cases}-\Delta u=\mu_{1}|u|^{p-2} u+\lambda \alpha|u|^{\alpha-2}|v|^{\beta} u & \text { in } \Omega, \\ -\Delta v=\mu_{2}|v|^{p-2} v+\lambda \beta|u|^{\alpha}|v|^{\beta-2} v & \text { in } \Omega, \\ u=v=0 & \text { on } \partial \Omega,\end{cases}
$$

has infinitely many fully nontrivial G-invariant solutions, one of which is positive.

For $p \in\left(2,2_{N}^{*}\right)$ this result is true without any symmetry assumption. For $p=2_{N}^{*}$ it was proved in [6, Corollary 1.3].

Taking $\mu_{1}=\mu_{2}=1$ and $\lambda=0$ in Theorem 1.1, we get a multiplicity result for problem (1.2). We shall prove, in fact, the following improvement of it that states the existence of infinitely many sign-changing solutions.

Theorem 1.3 Assume $\left(F_{1}\right)$ and $\left(F_{2}\right)$, and let $p \in\left(2,2_{N-d}^{*}\right)$. If $\Omega_{0} \neq \emptyset$, we also assume that $\gamma>\max \left\{\gamma_{p}, 0\right\}$. Then, the problem (1.2) has a positive $G$-invariant solution which has least energy among all nontrivial $G$-invariant solutions, and infinitely many sign-changing $G$-invariant solutions.

If $\Omega_{0}=\emptyset$ we may take $\gamma=0$, and (1.2) becomes

$$
-\Delta w=|w|^{p-2} w \text { in } \Omega, \quad w=0 \text { on } \partial \Omega .
$$

For this special case, Theorem 1.3 was proved in [8, Theorem 2.3]. The method that we will use to prove our results is an extension of the method used in [8].

If $\gamma>0$ and $\Omega_{0} \neq \emptyset$, then $\Omega \cap F \neq \emptyset$ and problem (1.2) is of Hénon-type. When $\Omega$ is the unit ball $B$ and $F=\{0\}$ it is the well-known Hénon problem

$$
-\Delta w=|x|^{\gamma}|w|^{p-2} w \text { in } B, \quad w=0 \text { on } \partial B,
$$

which has been widely studied, starting with the pioneering work [20] of W.-M. Ni, who proved the existence of a positive radial solution if $\gamma>\gamma_{p}$ or, equivalently, if $p<p_{\gamma}:=$ 
$\frac{2(N+\gamma)}{N-2}$. A Pohozaev-type identity shows that (1.4) does not have a nontrivial solution if $p \geq p_{\gamma} ;$ cf. Proposition 2.4.

Other special cases of Theorem 1.3 are given in [1,13]. In [1], Badiale and Serra established the existence of a positive $G$-invariant solution to (1.4) for the group $G=O(m) \times O(n)$, $m+n=N$, and $p$ and $\gamma$ as in Theorem 1.3. In [13], dos Santos and Pacella studied problem (1.4) when $N=2 m, G=O(m) \times O(m)$, and $F$ is either $\{0\}$ or $\mathbb{R}^{m} \times\{0\}$. For $p \in\left(2,2_{m+1}^{*}\right)$ and large enough $\gamma>0$, they established the existence of a positive least energy $G$-invariant solution which blows up at a $G$-orbit of minimal dimension in $\partial B \backslash F$ as $\gamma \rightarrow \infty$. We believe that a similar blow-up behavior is also true in our more general setting.

The Hénon problem (1.4) has also been studied in general bounded domains without any symmetries, and bubbling solutions have been constructed for exponents $p$ which are, either close to $2_{N}^{*}$, or slightly below the critical Hénon exponent $p_{\gamma}$. We refer to the recent papers $[2,12,14]$ for a detailed account.

As was shown in Conti et al. [11] for a subcritical system, the positive least energy solutions to the supercritical system (1.1) exhibit also phase separation as $\lambda \rightarrow-\infty$. More precisely, one has the following result.

Theorem 1.4 Assume that, for some sequence $\left(\lambda_{k}\right)$ with $\lambda_{k} \rightarrow-\infty$, there exists a positive fully nontrivial $G$-invariant solution $\left(u_{k}, v_{k}\right)$ to the system $(1.1)$ with $\lambda=\lambda_{k}$, which has least energy among all fully nontrivial $G$-invariant solutions to that system. Then, after passing to a subsequence, we have that

(a) $u_{k} \rightarrow u_{\infty}$ and $v_{k} \rightarrow v_{\infty}$ strongly in $D_{0}^{1,2}(\Omega)$,

(b) $u_{\infty}$ and $v_{\infty}$ are $G$-invariant, $u_{\infty} \geq 0, v_{\infty} \geq 0$ and $u_{\infty} v_{\infty} \equiv 0$,

(c) $u_{\infty}-v_{\infty}$ is a least energy $G$-invariant sign-changing solution to the problem

$$
\begin{cases}-\Delta w=\left|x_{2}\right|^{\gamma}\left(\mu_{1}\left|w^{+}\right|^{p-2} w^{+}+\mu_{2}\left|w^{-}\right|^{p-2} w^{-}\right) & \text {in } \Omega, \\ w=0 & \text { on } \partial \Omega,\end{cases}
$$

where $w^{+}:=\max \{w, 0\}$ and $w^{-}:=\min \{w, 0\}$.

This paper is organized as follows. In Sect. 2 we show that our assumptions on the symmetries yield a good variational setting for the system (1.1) and we discuss the variational problem. Section 3 is devoted to the proofs of Theorem 1.1 for $\lambda<0$ and Theorem 1.4. In Sect. 4 we prove Theorem 1.3 and we derive Theorem 1.1 for $\lambda>0$ from it.

\section{The symmetric variational setting}

Let $G$ be a closed subgroup of $O(N), F$ be a $G$-invariant linear subspace of $\mathbb{R}^{N}$ and $\Omega$ be a $G$-invariant bounded smooth domain in $\mathbb{R}^{N}$, which satisfy $\left(F_{1}\right)$ and $\left(F_{2}\right)$.

For $1 \leq p<\infty$ and $\gamma \geq 0$, we denote by

$$
L^{p}\left(\Omega ;\left|x_{2}\right|^{\gamma}\right):=\left\{w: \Omega \rightarrow \mathbb{R}:\left|x_{2}\right|^{\gamma / p} w \in L^{p}(\Omega)\right\}
$$

the weighted Lebesgue space with the norm given by

$$
|w|_{p, \gamma}:=\left(\int_{\Omega}\left|x_{2}\right|^{\gamma}|w|^{p}\right)^{1 / p} .
$$

Note that assumption $\left(F_{1}\right)$ guarantees that this is, indeed, a norm. As usual, we write $D_{0}^{1,2}(\Omega)$ for the closure of $\mathcal{C}_{c}^{\infty}(\Omega)$ in the Sobolev space $D^{1,2}\left(\mathbb{R}^{N}\right):=\left\{w \in L^{2^{*}}: \nabla w \in\right.$ 
$\left.L^{2}\left(\mathbb{R}^{N}, \mathbb{R}^{N}\right)\right\}$, equipped with the norm

$$
\|w\|:=\left(\int_{\mathbb{R}^{N}}|\nabla w|^{2}\right)^{1 / 2}
$$

A (weak) solution to the system (1.1) is a pair $(u, v)$ such that $u, v \in D_{0}^{1,2}(\Omega) \cap$ $L^{p}\left(\Omega ;\left|x_{2}\right|^{\gamma}\right)$, and they satisfy the identities

$$
\begin{aligned}
& \int_{\Omega} \nabla u \cdot \nabla \vartheta-\mu_{1} \int_{\Omega}\left|x_{2}\right|^{\gamma}|u|^{p-2} u \vartheta-\lambda \alpha \int_{\Omega}\left|x_{2}\right|^{\gamma}|u|^{\alpha-2} u|v|^{\beta} \vartheta=0, \\
& \int_{\Omega} \nabla v \cdot \nabla \vartheta-\mu_{2} \int_{\Omega}\left|x_{2}\right|^{\gamma}|v|^{p-2} v \vartheta-\lambda \beta \int_{\Omega}\left|x_{2}\right|^{\gamma}|u|^{\alpha}|v|^{\beta-2} v \vartheta=0,
\end{aligned}
$$

for every $\vartheta \in \mathcal{C}_{c}^{\infty}(\Omega)$.

Hebey and Vaugon showed in [16] that the Sobolev embedding and the RellichKondrachov theorems can be improved in $G$-invariant domains whose $G$-orbits have positive dimension. Ivanov and Nazarov obtained an extension of this result in [17], which allows to consider domains with finite $G$-orbits. These results will play a crucial role in the proof of our main result. The version that we need will be derived from them next.

Set

$$
D_{0}^{1,2}(\Omega)^{G}:=\left\{w \in D_{0}^{1,2}(\Omega): w \text { is } G \text {-invariant }\right\},
$$

and recall the definitions of $\Omega_{0}:=\{x \in \Omega: \operatorname{dim} G x=0\}$,

$$
d:=\min \left\{\operatorname{dim} G x: x \in \Omega \backslash \Omega_{0}\right\}, \quad \gamma_{p}:=p\left(\frac{N}{2}-\frac{N}{p}-1\right),
$$

$2_{k}^{*}:=\frac{2 k}{k-2}$ if $k>2$ and $2_{k}^{*}:=\infty$ if $k \leq 2$.

Theorem 2.1 (Hebey-Vaugon [16]; Ivanov-Nazarov [17]) If $p \in \mathbb{R} \cap\left[1,2_{N-d}^{*}\right]$ and $\gamma \geq$ $\max \left\{\gamma_{p}, 0\right\}$, then the embedding

$$
D_{0}^{1,2}(\Omega)^{G} \hookrightarrow L^{p}\left(\Omega ;\left|x_{2}\right|^{\gamma}\right)
$$

is continuous. Moreover, this embedding is compact if $p \in\left[1,2_{N-d}^{*}\right.$ ) and, either $\Omega_{0}=\emptyset$, or $\Omega_{0} \neq \emptyset$ and $\gamma>\max \left\{\gamma_{p}, 0\right\}$.

Proof If $\Omega_{0}=\emptyset$ and $\gamma=0$, this statement is proved in [16, Corollary 2]. The result for $\Omega_{0}=\emptyset$ and $\gamma>0$ follows immediately from it.

If $\Omega_{0} \neq \emptyset$ and $\gamma>\max \left\{\gamma_{p}, 0\right\}$, let $\mathfrak{r}(x)$ denote the Riemannian distance in $\Omega$ from $x$ to $\Omega_{0}$. The statement of this theorem with $L^{p}\left(\Omega ;\left|x_{2}\right|^{\gamma}\right)$ replaced by $L^{p}\left(\Omega ; \mathfrak{r}(x)^{\gamma}\right)$ was proved in [17, Theorem 2.4]. Since assumption $\left(F_{2}\right)$ implies that $\left|x_{2}\right| \leq \mathfrak{r}(x)$ for every $x \in \Omega$, our claim follows.

From now on we will assume that $p \in\left(2,2_{N-d}^{*}\right)$ and that $\gamma>\max \left\{\gamma_{p}, 0\right\}$ if $\Omega_{0} \neq \emptyset$. We write

$$
S_{p, \gamma}^{G}:=\inf _{\substack{u \in D_{0}^{1,2}(\Omega)^{G} \\ u \neq 0}} \frac{\|u\|^{2}}{|u|_{p, \gamma}^{2}}
$$

for the best constant for the embedding $D_{0}^{1,2}(\Omega)^{G} \hookrightarrow L^{p}\left(\Omega ;\left|x_{2}\right|^{\gamma}\right)$. 
Let $\mathscr{D}^{G}:=D_{0}^{1,2}(\Omega)^{G} \times D_{0}^{1,2}(\Omega)^{G}$. Theorem 2.1 guarantees that the functional $E: \mathscr{D}^{G} \rightarrow$ $\mathbb{R}$, given by

$$
\begin{aligned}
E(u, v):= & \frac{1}{2} \int_{\Omega}\left(|\nabla u|^{2}+|\nabla v|^{2}\right)-\frac{1}{p} \int_{\Omega}\left|x_{2}\right|^{\gamma}\left(\mu_{1}|u|^{p}+\mu_{2}|v|^{p}\right) \\
& -\lambda \int_{\Omega}\left|x_{2}\right|^{\gamma}|u|^{\alpha}|v|^{\beta}
\end{aligned}
$$

is well defined. It is of class $\mathcal{C}^{1}$ and has the following property.

Proposition 2.2 The critical points of $E: \mathscr{D}^{G} \rightarrow \mathbb{R}$ are the $G$-invariant solutions to the system (1.1).

Proof Let $(u, v)$ be a critical point of $E: \mathscr{D}^{G} \rightarrow \mathbb{R}$. Then,

$$
\begin{aligned}
& \partial_{u} E(u, v) \varphi=\int_{\Omega} \nabla u \cdot \nabla \varphi-\mu_{1} \int_{\Omega}\left|x_{2}\right|^{\gamma}|u|^{p-2} u \varphi-\lambda \alpha \int_{\Omega}\left|x_{2}\right|^{\gamma}|u|^{\alpha-2} u|v|^{\beta} \varphi=0, \\
& \partial_{v} E(u, v) \varphi=\int_{\Omega} \nabla v \cdot \nabla \varphi-\mu_{2} \int_{\Omega}\left|x_{2}\right|^{\gamma}|v|^{p-2} v \varphi-\lambda \beta \int_{\Omega}\left|x_{2}\right|^{\gamma}|u|^{\alpha}|v|^{\beta-2} v \varphi=0,
\end{aligned}
$$

for every $G$-invariant function $\varphi \in \mathcal{C}_{c}^{\infty}(\Omega)$.

By Theorem 2.1 we have that $u, v \in L^{p}\left(\Omega ;\left|x_{2}\right|^{\gamma}\right)$. So we need only to prove that the identities (2.1) and (2.2) hold true for every $\vartheta \in \mathcal{C}_{c}^{\infty}(\Omega)$.

Let $\vartheta \in \mathcal{C}_{c}^{\infty}(\Omega)$, and define

$$
\varphi(x):=\frac{1}{\mu(G)} \int_{G} \vartheta(g x) \mathrm{d} \mu,
$$

where $\mu$ is the Haar measure on $G$; see [19]. Then, $\varphi$ is $G$-invariant. A straightforward computation yields

$$
\begin{aligned}
& 0=\partial_{u} E(u, v) \varphi=\int_{\Omega} \nabla u \cdot \nabla \vartheta-\mu_{1} \int_{\Omega}\left|x_{2}\right|^{\gamma}|u|^{p-2} u \vartheta-\lambda \alpha \int_{\Omega}\left|x_{2}\right|^{\gamma}|u|^{\alpha-2} u|v|^{\beta} \vartheta, \\
& 0=\partial_{v} E(u, v) \varphi=\int_{\Omega} \nabla v \cdot \nabla \vartheta-\mu_{2} \int_{\Omega}\left|x_{2}\right|^{\gamma}|v|^{p-2} v \vartheta-\lambda \beta \int_{\Omega}\left|x_{2}\right|^{\gamma}|u|^{\alpha}|v|^{\beta-2} v \vartheta,
\end{aligned}
$$

cf. [10, Lemma 2.1]. This completes the proof.

We define

$$
\begin{aligned}
& f(u, v):=\partial_{u} E(u, v) u=\int_{\Omega}|\nabla u|^{2}-\mu_{1} \int_{\Omega}\left|x_{2}\right|^{\gamma}|u|^{p}-\lambda \alpha \int_{\Omega}\left|x_{2}\right|^{\gamma}|u|^{\alpha}|v|^{\beta}, \\
& h(u, v):=\partial_{v} E(u, v) v=\int_{\Omega}|\nabla v|^{2}-\mu_{2} \int_{\Omega}\left|x_{2}\right|^{\gamma}|v|^{p}-\lambda \beta \int_{\Omega}\left|x_{2}\right|^{\gamma}|u|^{\alpha}|v|^{\beta},
\end{aligned}
$$

and consider the set

$$
\mathcal{N}^{G}:=\left\{(u, v) \in \mathscr{D}^{G}: u \neq 0, v \neq 0, f(u, v)=h(u, v)=0\right\} .
$$

The following proposition improves [9, Proposition 2.1], as it allows $\alpha, \beta>2$.

Proposition 2.3 If $\lambda<0$, the following statements hold true:

(a) There exists $c_{0}>0$ such that, for every $(u, v) \in \mathcal{N}^{G}$,

$$
c_{0} \leq\|u\|^{2} \leq \mu_{1}|u|_{p, \gamma}^{p} \text { and } c_{0} \leq\|v\|^{2} \leq \mu_{2}|v|_{p, \gamma}^{p} .
$$


(b) $\mathcal{N}^{G}$ is a closed $\mathcal{C}^{1}$-submanifold of codimension 2 of $\mathscr{D}^{G}$. More precisely, $\nabla f(u, v)$ and $\nabla h(u, v)$ are linearly independent and generate the orthogonal complement of the tangent space to $\mathcal{N}^{G}$ at $(u, v)$.

(c) $\mathcal{N}^{G}$ is a natural constraint for $E: \mathscr{D}^{G} \rightarrow \mathbb{R}$, i.e., the critical points of the restriction of $E$ to $\mathcal{N}^{G}$ are critical points of $E$.

Proof (a) : Let $(u, v) \in \mathcal{N}^{G}$. Then, as $\lambda<0$, we have that $\|u\|^{2} \leq \mu_{1}|u|_{p, \gamma}^{p}$. Therefore,

$$
0<S_{p, \gamma}^{G} \leq \frac{\|u\|^{2}}{|u|_{p, \gamma}^{2}} \leq \mu_{1}^{2 / p}\left(\|u\|^{2}\right)^{(p-2) / p} .
$$

Multiplying this inequality by $\mu_{1}^{-2 / p}$ and raising it to the power of $p /(p-2)$, we obtain the statement for $u$. An analogous argument can be used for $v$.

(b) : First, notice that $\mathcal{N}^{G} \neq \varnothing$. Indeed, if $\varphi_{1}, \varphi_{2} \in \mathcal{C}_{c}^{\infty}(\Omega)$ are nontrivial $G$-invariant functions with disjoint supports and $s_{1}, s_{2}>0$ are such that $\left\|s_{i} \varphi_{i}\right\|^{2}=\mu_{i}\left|s_{i} \varphi_{i}\right|_{p, \gamma}^{p}$, then $\left(s_{1} \varphi_{1}, s_{2} \varphi_{2}\right) \in \mathcal{N}^{G}$.

Statement (a) implies that $\mathcal{N}^{G}$ is closed in $\mathscr{D}^{G}$. Next, we prove that $\nabla f(u, v)$ and $\nabla h(u, v)$ are linearly independent if $(u, v) \in \mathcal{N}^{G}$. Assume there exist $s, t \in \mathbb{R}$ such that $s \nabla f(u, v)+$ $t \nabla h(u, v)=0$. Taking the scalar product of this expression with $(u, 0)$, and using the fact that $f(u, v)=0=h(u, v)$, we obtain

$$
\begin{aligned}
0= & s\langle\nabla f(u, v),(u, 0)\rangle+t\langle\nabla h(u, v),(u, 0)\rangle \\
= & s\left((2-p) \mu_{1} \int_{\Omega}\left|x_{2}\right|^{\gamma}|u|^{p}+\lambda \alpha(2-\alpha) \int_{\Omega}\left|x_{2}\right|^{\gamma}|u|^{\alpha}|v|^{\beta}\right) \\
& +t\left(-\lambda \alpha \beta \int_{\Omega}\left|x_{2}\right|^{\gamma}|u|^{\alpha}|v|^{\beta}\right) \\
= & : s a_{11}+t a_{12} .
\end{aligned}
$$

Similarly, taking the scalar product with $(0, v)$ yields

$$
\begin{aligned}
0= & s\langle\nabla f(u, v),(0, v)\rangle+t\langle\nabla h(u, v),(0, v)\rangle \\
= & s\left(-\lambda \alpha \beta \int_{\Omega}\left|x_{2}\right|^{\gamma}|u|^{\alpha}|v|^{\beta}\right) \\
& +t\left((2-p) \mu_{2} \int_{\Omega}\left|x_{2}\right|^{\gamma}|v|^{p}+\lambda \beta(2-\beta) \int_{\Omega}\left|x_{2}\right|^{\gamma}|u|^{\alpha}|v|^{\beta}\right) \\
= & : s a_{21}+t a_{22} .
\end{aligned}
$$

We consider two cases. First, if $\int_{\Omega}\left|x_{2}\right|^{\gamma}|u|^{\alpha}|v|^{\beta}=0$, statement $(a$ ) yields

$$
\operatorname{det}\left(a_{i j}\right)=(2-p)^{2}\left(\mu_{1}|u|_{p, \gamma}^{p}\right)\left(\mu_{2}|v|_{p, \gamma}^{p}\right) \geq(2-p)^{2} c_{0}^{2}>0 .
$$

On the other hand, if $\int_{\Omega}\left|x_{2}\right|^{\gamma}|u|^{\alpha}|v|^{\beta} \neq 0$, then, as $\lambda<0$ and $\alpha, \beta<p$,

$$
\begin{aligned}
& A:=\frac{\mu_{1} \int_{\Omega}\left|x_{2}\right|^{\gamma}|u|^{p}}{-\lambda \int_{\Omega}\left|x_{2}\right|^{\gamma}|u|^{\alpha}|v|^{\beta}} \geq\left(\frac{c_{0}}{-\lambda p \int_{\Omega}\left|x_{2}\right|^{\gamma}|u|^{\alpha}|v|^{\beta}}+1\right) \alpha, \\
& B:=\frac{\mu_{2} \int_{\Omega}\left|x_{2}\right|^{\gamma}|v|^{p}}{-\lambda \int_{\Omega}\left|x_{2}\right|^{\gamma}|u|^{\alpha}|v|^{\beta}} \geq\left(\frac{c_{0}}{-\lambda p \int_{\Omega}\left|x_{2}\right|^{\gamma}|u|^{\alpha}|v|^{\beta}}+1\right) \beta .
\end{aligned}
$$


Note that

$$
\operatorname{det}\left(a_{i j}\right)=\left(\lambda \int_{\Omega}\left|x_{2}\right|^{\gamma}|u|^{\alpha}|v|^{\beta}\right)^{2}\left|\begin{array}{cc}
(2-p) A-\alpha(2-\alpha) & \alpha \beta \\
\alpha \beta & (2-p) B-\beta(2-\beta)
\end{array}\right|
$$

and

$$
\begin{aligned}
\left|\begin{array}{cc}
(2-p) A-\alpha(2-\alpha) & \alpha \beta \\
\alpha \beta & (2-p) B-\beta(2-\beta)
\end{array}\right| \\
\quad=(2-p)((2-p) A B-[\beta(2-\beta) A+\alpha(2-\alpha) B]+2 \alpha \beta) \\
\quad=(p-2) \alpha \beta\left((p-2) \frac{A}{\alpha} \frac{B}{\beta}-(\beta-2) \frac{A}{\alpha}-(\alpha-2) \frac{B}{\beta}-2\right) .
\end{aligned}
$$

Next, we show that there is a constant $c_{1}>0$ such that

$$
D:=(p-2) \frac{A}{\alpha} \frac{B}{\beta}-(\beta-2) \frac{A}{\alpha}-(\alpha-2) \frac{B}{\beta}-2>\frac{c_{1}}{-\lambda \int_{\Omega}\left|x_{2}\right|^{\gamma}|u|^{\alpha}|v|^{\beta}} .
$$

To prove this inequality, we consider two cases. Recall that $\alpha<A, \beta<B$ and $\alpha+\beta=p>2$.

(i) If $\alpha, \beta<2$, then $D>(p-2) \frac{A}{\alpha}-(\beta-2)-(\alpha-2)-2=(p-2)\left(\frac{A}{\alpha}-1\right)$.

(ii) If $\alpha \geq 2$, then

$$
D>(p-2) \frac{A}{\alpha} \frac{B}{\beta}-\beta \frac{A}{\alpha} \frac{B}{\beta}-(\alpha-2) \frac{A}{\alpha} \frac{B}{\beta}+2\left(\frac{A}{\alpha}-1\right)=2\left(\frac{A}{\alpha}-1\right) .
$$

Similarly, $D>2\left(\frac{B}{\beta}-1\right)$ if $\beta \geq 2$.

Combining these inequalities with (2.3) and (2.4), we obtain (2.5). Consequently,

$$
\operatorname{det}\left(a_{i j}\right)>c_{2}\left(\int_{\Omega}\left|x_{2}\right|^{\gamma}|u|^{\alpha}|v|^{\beta}\right),
$$

for some constant $c_{2}>0$. So, in any case, $\operatorname{det}\left(a_{i j}\right)>0$ and, therefore, $\nabla f(u, v)$ and $\nabla h(u, v)$ are linearly independent, as claimed.

(c) : If $(u, v) \in \mathcal{N}^{G}$ is a critical point of the restriction $\left.E\right|_{\mathcal{N}^{G}}$, then

$$
\nabla E(u, v)=s \nabla f(u, v)+t \nabla h(u, v) \text { for some } s, t \in \mathbb{R} .
$$

Taking the scalar product with $(u, 0)$ and $(0, v)$, we obtain

$$
\begin{aligned}
& s\langle\nabla f(u, v),(u, 0)\rangle+t\langle\nabla h(u, v),(u, 0)\rangle=\langle\nabla E(u, v),(u, 0)\rangle=f(u, v)=0, \\
& s\langle\nabla f(u, v),(0, v)\rangle+t\langle\nabla h(u, v),(0, v)\rangle=\langle\nabla E(u, v),(0, v)\rangle=h(u, v)=0 .
\end{aligned}
$$

But, as was seen in statement $(b)$, this happens only if $s=t=0$. Hence, $\nabla E(u, v)=0$, as claimed.

We end this section with the following nonexistence result.

Proposition 2.4 If $\Omega$ is star shaped with respect to the origin and $p \geq p_{\gamma}:=\frac{2(N+\gamma)}{N-2}$, the system (1.1) does not have a nontrivial solution.

Proof If $(u, v)$ is a solution to (1.1), then the identity in [24, Proposition 3] with $\mathscr{F}(x, u, v, y, z):=\frac{1}{2}\left(|y|^{2}+|z|^{2}\right)-\frac{1}{p}\left|x_{2}\right|^{\gamma}\left(\mu_{1}|u|^{p}+\mu_{2}|v|^{p}\right)-\lambda\left|x_{2}\right|^{\gamma}|u|^{\alpha}|v|^{\beta}, h(x):=x$ 
and $a:=0, x \in \Omega, u, v \in \mathbb{R}, y, z \in \mathbb{R}^{N}$, holds true. Integrating this identity over $\Omega$ and noting that $f(u, v)=0=h(u, v)$ we get

$$
\begin{aligned}
-\frac{1}{2} \int_{\partial \Omega} & \left(\left|\frac{\partial u}{\partial v}\right|^{2}+\left|\frac{\partial v}{\partial v}\right|^{2}\right)(x \cdot v) \\
= & \frac{N-2}{2} \int_{\Omega}\left(|\nabla u|^{2}+|\nabla v|^{2}\right)-\frac{N+\gamma}{p} \int_{\Omega}\left|x_{2}\right|^{\gamma}\left(\mu_{1}|u|^{p}+\mu_{2}|v|^{p}\right) \\
& -\lambda(N+\gamma) \int_{\Omega}\left|x_{2}\right|^{\gamma}|u|^{\alpha}|v|^{\beta} \\
= & \left(\frac{N-2}{2}-\frac{N+\gamma}{p}\right) \int_{\Omega}\left(|\nabla u|^{2}+|\nabla v|^{2}\right) .
\end{aligned}
$$

Observe that $\frac{N-2}{2} \geq \frac{N+\gamma}{p}$ iff $p \geq p_{\gamma}$. So the result follows immediately from this identity if $p>p_{\gamma}$. If $p=p_{\gamma}$, we apply the unique continuation principle.

\section{The competitive system}

Throughout this section we assume that $\lambda<0$. We continue to assume that $p \in\left(2,2_{N-d}^{*}\right)$ and that $\gamma>\max \left\{\gamma_{p}, 0\right\}$ if $\Omega_{0} \neq \emptyset$.

Our first goal is to prove Theorem 1.1 for $\lambda<0$. Using a critical point result due to Szulkin [26], we will show that the functional $E$ restricted to $\mathcal{N}^{G}$ has infinitely many critical points. By Propositions 2.2 and 2.3, they are fully nontrivial solutions to the system (1.1).

We write $\nabla_{\mathcal{N}^{G}} E(u, v)$ for the orthogonal projection of $\nabla E(u, v)$ onto the tangent space to $\mathcal{N}^{G}$ at $(u, v)$.

The proofs of the following two lemmas are similar to those of the analogous statements in [9]. We include them here for the sake of completeness.

Lemma 3.1 The functional $E: \mathcal{N}^{G} \rightarrow \mathbb{R}$ satisfies the Palais-Smale condition $(\mathrm{PS})_{c}$ for every $c \in \mathbb{R}$, i.e., every sequence $\left(\left(u_{k}, v_{k}\right)\right)$ in $\mathcal{N}^{G}$ such that

$$
E\left(u_{k}, v_{k}\right) \rightarrow c \text { and } \nabla_{\mathcal{N}^{G}} E(u, v) \rightarrow 0
$$

contains a subsequence which converges strongly in $\mathscr{D}$.

Proof Let $\left(\left(u_{k}, v_{k}\right)\right)$ be as above. It is easy to see that the sequences $\left(\left(u_{k}, v_{k}\right)\right),\left(\nabla f\left(u_{k}, v_{k}\right)\right)$ and $\left(\nabla h\left(u_{k}, v_{k}\right)\right)$ are bounded in $\mathscr{D}^{G}$.

We claim that $\nabla E\left(u_{k}, v_{k}\right) \rightarrow 0$. To prove this claim, we write

$$
\nabla E\left(u_{k}, v_{k}\right)=\nabla_{\mathcal{N}^{G}} E\left(u_{k}, v_{k}\right)+s_{k} \nabla f\left(u_{k}\right)+t_{k} \nabla h\left(u_{k}\right)
$$

with $s_{k}, t_{k} \in \mathbb{R}$. Taking the scalar product of this identity with $\left(u_{k}, 0\right)$ and $\left(0, v_{k}\right)$, we see that $s_{k}$ and $t_{k}$ solve the system

$$
\left\{\begin{array}{l}
o(1)=s_{k} a_{11}^{(k)}+t_{k} a_{12}^{(k)}, \\
o(1)=s_{k} a_{21}^{(k)}+t_{k} a_{22}^{(k)}
\end{array}\right.
$$


where $o(1) \rightarrow 0$ as $k \rightarrow \infty$,

$$
\begin{aligned}
& a_{11}^{(k)}:=(2-p) \mu_{1} \int_{\Omega}\left|x_{2}\right|^{\gamma}\left|u_{k}\right|^{p}+\lambda \alpha(2-\alpha) \int_{\Omega}\left|x_{2}\right|^{\gamma}\left|u_{k}\right|^{\alpha}\left|v_{k}\right|^{\beta}, \\
& a_{12}^{(k)}:=-\lambda \alpha \beta \int_{\Omega}\left|x_{2}\right|^{\gamma}\left|u_{k}\right|^{\alpha}\left|v_{k}\right|^{\beta}=: a_{21}^{(k)}, \\
& a_{22}^{(k)}:=(2-p) \mu_{2} \int_{\Omega}\left|x_{2}\right|^{\gamma}\left|v_{k}\right|^{p}+\lambda \beta(2-\beta) \int_{\Omega}\left|x_{2}\right|^{\gamma}\left|u_{k}\right|^{\alpha}\left|v_{k}\right|^{\beta} .
\end{aligned}
$$

After passing to a subsequence, we have that $\int_{\Omega}\left|x_{2}\right|^{\gamma}\left|u_{k}\right|^{\alpha}\left|v_{k}\right|^{\beta} \rightarrow b \in[0, \infty)$. If $b=0$, Proposition 2.3( $a$ ) implies that

$$
\operatorname{det}\left(a_{i j}^{(k)}\right) \geq \frac{1}{2}(2-p)^{2} c_{0}^{2} \quad \text { for } k \text { large enough. }
$$

If $b \neq 0$, statement (2.6) yields

$$
\operatorname{det}\left(a_{i j}^{(k)}\right) \geq \frac{c_{2}}{2} b>0 \quad \text { for } k \text { large enough. }
$$

Hence, after passing to a subsequence, we have that $s_{k} \rightarrow 0$ and $t_{k} \rightarrow 0$, and from (3.1) we get that $\nabla E\left(u_{k}, v_{k}\right) \rightarrow 0$, as claimed.

By Theorem 2.1, the embedding $\mathscr{D}^{G} \hookrightarrow L^{p}\left(\Omega ;\left|x_{2}\right|^{\gamma}\right) \times L^{p}\left(\Omega ;\left|x_{2}\right|^{\gamma}\right)$ is compact. Using this fact, it is now standard to show that $\left(\left(u_{k}, v_{k}\right)\right)$ contains a subsequence which converges strongly in $\mathscr{D}^{G}$.

Let $Z$ be a symmetric subset of $\mathcal{N}^{G}$, i.e., $(-u,-v) \in \mathcal{N}^{G}$ iff $(u, v) \in \mathcal{N}^{G}$. If $Z \neq \emptyset$, the genus of $Z$ is the smallest integer $j \geq 1$ such that there exists an odd continuous function $Z \rightarrow \mathbb{S}^{j-1}$ into the unit sphere $\mathbb{S}^{j-1}$ in $\mathbb{R}^{j}$. We denote it by genus( $Z$ ). If no such $j$ exists, we define $\operatorname{genus}(Z):=\infty$. We set genus $(\emptyset):=0$.

Lemma 3.2 For all $j \geq 1$,

$$
\Sigma_{j}:=\left\{Z \subset \mathcal{N}^{G}: Z \text { is symmetric and compact, and genus }(Z) \geq j\right\} \neq \emptyset .
$$

Proof If $u, v \in D_{0}^{1,2}(\Omega)^{G}, u, v \neq 0$, we denote by $s_{u}, t_{v}$ the unique positive numbers such that $\left\|s_{u} u\right\|^{2}=\mu_{1}\left|s_{u} u\right|_{p, \gamma}^{p}$ and $\left\|t_{v} v\right\|^{2}=\mu_{2}\left|t_{v} v\right|_{p, \gamma}^{p}$. Note that $\left(s_{u} u, t_{v} v\right) \in \mathcal{N}^{G}$ if $u v=0$. We write

$$
\rho(u, v):=\left(s_{u} u, t_{v} v\right) .
$$

Given $j \geq 1$, we choose $G$-invariant functions $u_{1}, \ldots, u_{j}, v_{1}, \ldots, v_{j}$ in $\mathcal{C}_{c}^{\infty}(\Omega)$ such that any two of them have disjoint supports. Let $\left\{e_{i}: 1 \leq i \leq j\right\}$ be the canonical basis of $\mathbb{R}^{j}$, and $Q$ be the convex hull of $\left\{ \pm e_{i}: 1 \leq i \leq j\right\}$, i.e.,

$$
Q:=\left\{\sum_{i=1}^{j} r_{i} \hat{e}_{i}: \hat{e}_{i} \in\left\{ \pm e_{i}\right\}, r_{i} \in[0,1], \sum_{i=1}^{j} r_{i}=1\right\},
$$

which is homeomorphic to the unit sphere $\mathbb{S}^{j-1}$ in $\mathbb{R}^{j}$ by an odd homeomorphism.

We define $\sigma: Q \rightarrow \mathcal{N}^{\Gamma}$ by setting $\sigma\left(e_{i}\right):=\left(u_{i}, v_{i}\right), \sigma\left(-e_{i}\right):=\left(-u_{i},-v_{i}\right)$, and

$$
\sigma\left(\sum_{i=1}^{j} r_{i} \hat{e}_{i}\right):=\rho\left(\sum_{i=1}^{j} r_{i} \sigma\left(\hat{e}_{i}\right)\right) .
$$


This is a well defined, continuous, odd map. Hence, $Z:=\sigma(Q)$ is a symmetric compact subset of $\mathcal{N}^{G}$. If $\tau: Z \rightarrow \mathbb{S}^{k-1}$ is an odd continuous map, the composition $\tau \circ \sigma$ yields an odd continuous map $\mathbb{S}^{j-1} \rightarrow \mathbb{S}^{k-1}$, which, by the Borsuk-Ulam theorem, forces $k \geq j$. This shows that genus $(Z) \geq j$. Thus, $Z \in \Sigma_{j}$.

Proof of Theorem 1.1 for $\lambda<0$. By Proposition 2.3 and Lemma 3.1, $\mathcal{N}^{G}$ is a closed symmetric $\mathcal{C}^{1}$-submanifold of $\mathscr{D}^{G}$ that does not contain the origin, and $E$ is an even $\mathcal{C}^{1}$ functional, which is bounded below on $\mathcal{N}^{G}$ by a positive constant and satisfies (PS) $)_{c}$ for every $c \in \mathbb{R}$. Since, by Lemma 3.2, $\Sigma_{j} \neq \varnothing$ for every $j \geq 1$, Szulkin's multiplicity result [26, Corollary 4.1] implies that $E$ attains its minimum and has infinitely many critical points on $\mathcal{N}^{G}$. As $E(u, v)=E(|u|,|v|)$, the minimum can be chosen to be positive.

To prove Theorem 1.4, we could follow the argument of [9, Proposition 5.1]. A simpler argument is given next. To highlight the role played by $\lambda$, we write $E_{\lambda}, f_{\lambda}, h_{\lambda}, \mathcal{N}_{\lambda}^{G}$ instead of $E, f, h, \mathcal{N}^{G}$, and set

$$
c_{\lambda}^{G}:=\inf _{(u, v) \in \mathcal{N}_{\lambda}^{G}} E_{\lambda}(u, v)
$$

The nontrivial $G$-invariant solutions to the problem (1.5) are the critical points of the restriction of the functional $J: D_{0}^{1,2}(\Omega)^{G} \rightarrow \mathbb{R}$ to the Nehari manifold $\mathcal{M}^{G}$, defined as

$$
\begin{aligned}
J(w) & :=\frac{1}{2} \int_{\Omega}|\nabla w|^{2}-\frac{1}{p} \int_{\Omega}\left|x_{2}\right|^{\gamma}\left(\mu_{1}\left|w^{+}\right|^{p}+\mu_{2}\left|w^{-}\right|^{p}\right), \\
\mathcal{M}^{G} & :=\left\{w \in D_{0}^{1,2}(\Omega)^{G}: w \neq 0, \int_{\Omega}|\nabla w|^{2}=\int_{\Omega}\left|x_{2}\right|^{\gamma}\left(\mu_{1}\left|w^{+}\right|^{p}+\mu_{2}\left|w^{-}\right|^{p}\right)\right\} .
\end{aligned}
$$

The sign-changing solutions lie on the set

$$
\mathcal{E}^{G}:=\left\{w \in D_{0}^{1,2}(\Omega)^{G}: w^{+}, w^{-} \in \mathcal{M}^{G}\right\} .
$$

We define

$$
c_{\infty}^{G}:=\inf _{w \in \mathcal{E}^{G}} J(w)
$$

Note that if $w \in \mathcal{E}^{G}$, then, as $w^{+} w^{-}=0$, we have that $J(w)=E\left(w^{+}, w^{-}\right)$and $\left(w^{+}, w^{-}\right) \in$ $\mathcal{N}_{\lambda}^{G}$. Therefore, $c_{\lambda}^{G} \leq c_{\infty}^{G}$ for every $\lambda<0$.

Proof of Theorem 1.4 Let $\lambda_{k} \rightarrow-\infty$ and $\left(u_{k}, v_{k}\right) \in \mathcal{N}_{\lambda_{k}}^{G}$ satisfy $E\left(u_{k}, v_{k}\right)=c_{\lambda_{k}}^{G}$ and $u_{k}, v_{k} \geq 0$. Then,

$$
\frac{p-2}{2 p} \int_{\Omega}\left(\left|\nabla u_{k}\right|^{2}+\left|\nabla v_{k}\right|^{2}\right)=c_{\lambda_{k}}^{G} \leq c_{\infty}^{G} .
$$

So, after passing to a subsequence,

$$
\begin{aligned}
& u_{k} \rightarrow u_{\infty}, \quad v_{k} \rightarrow v_{\infty}, \quad \text { weakly in } D_{0}^{1,2}(\Omega)^{G}, \\
& u_{k} \rightarrow u_{\infty}, \quad v_{k} \rightarrow v_{\infty}, \quad \text { strongly in } L^{p}\left(\Omega ;\left|x_{2}\right|^{\gamma}\right), \\
& u_{k} \rightarrow u_{\infty}, \quad v_{k} \rightarrow v_{\infty}, \quad \text { a.e. in } \Omega \text {. }
\end{aligned}
$$

Hence, $u_{\infty}, v_{\infty} \geq 0$. Moreover, as $f_{\lambda_{k}}\left(u_{k}, v_{k}\right)+h_{\lambda_{k}}\left(u_{k}, v_{k}\right)=0$, we have that

$$
0 \leq \int_{\Omega}\left|x_{2}\right|^{\gamma}\left|u_{k}\right|^{\alpha}\left|v_{k}\right|^{\beta} \leq \frac{1}{p\left(-\lambda_{k}\right)} \int_{\Omega}\left|x_{2}\right|^{\gamma}\left(\mu_{1}\left|u_{k}\right|^{p}+\mu_{2}\left|v_{k}\right|^{p}\right) \leq \frac{C_{0}}{\left(-\lambda_{k}\right)},
$$


and from Fatou's lemma we obtain

$$
0 \leq \int_{\Omega}\left|x_{2}\right|^{\gamma}\left|u_{\infty}\right|^{\alpha}\left|v_{\infty}\right|^{\beta} \leq \lim _{k \rightarrow \infty} \int_{\Omega}\left|x_{2}\right|^{\gamma}\left|u_{k}\right|^{\alpha}\left|v_{k}\right|^{\beta}=0 .
$$

Hence, $u_{\infty} v_{\infty}=0$ a.e. in $\Omega$. On the other hand, by Proposition 2.3,

$$
0<c_{0} \leq\left\|u_{k}\right\|^{2} \leq \mu_{1}\left|u_{k}\right|_{p, \gamma}^{p} \quad \text { and } \quad 0<c_{0} \leq\left\|v_{k}\right\|^{2} \leq \mu_{2}\left|v_{k}\right|_{p, \gamma}^{p} .
$$

So, passing to the limit, we obtain that $u_{\infty} \neq 0$ and $v_{\infty} \neq 0$. Moreover,

$$
\left\|u_{\infty}\right\|^{2} \leq \mu_{1}\left|u_{\infty}\right|_{p, \gamma}^{p} \quad \text { and } \quad\left\|v_{\infty}\right\|^{2} \leq \mu_{2}\left|v_{\infty}\right|_{p, \gamma}^{p} .
$$

Let $s, t$ be the unique positive numbers such that $\left\|s u_{\infty}\right\|^{2}=\mu_{1}\left|s u_{\infty}\right|_{p, \gamma}^{p}$ and $\left\|t v_{\infty}\right\|^{2}=$ $\mu_{2}\left|t v_{\infty}\right|_{p, \gamma}^{p}$. Then, $s u_{\infty}-t v_{\infty} \in \mathcal{E}^{G}$. The inequalities (3.4) imply that $s, t \in(0,1]$. Therefore,

$$
\begin{aligned}
c_{\infty}^{G} & \leq \frac{p-2}{2 p}\left(\left\|s u_{\infty}\right\|^{2}+\left\|t v_{\infty}\right\|^{2}\right) \leq \frac{p-2}{2 p}\left(\left\|u_{\infty}\right\|^{2}+\left\|v_{\infty}\right\|^{2}\right) \\
& \leq \frac{p-2}{2 p} \lim _{k \rightarrow \infty}\left(\left\|u_{k}\right\|^{2}+\left\|v_{k}\right\|^{2}\right)=\lim _{k \rightarrow \infty} c_{\lambda_{k}}^{G} \leq c_{\infty}^{G} .
\end{aligned}
$$

It follows that $u_{k} \rightarrow u_{\infty}$ and $v_{k} \rightarrow v_{\infty}$ strongly in $D_{0}^{1,2}(\Omega)^{G}, s=t=1, u_{\infty}-v_{\infty} \in \mathcal{E}^{G}$ and $J\left(u_{\infty}-v_{\infty}\right)=c_{\infty}^{G}$. Arguing as in [3, Lemma 2.6] we conclude that $u_{\infty}-v_{\infty}$ is a least energy $G$-invariant sign-changing solution to the problem (1.5).

\section{The equation and the cooperative system}

First, we prove Theorem 1.3.

Proof of Theorem 1.3 The nontrivial $G$-invariant solutions to the problem (1.2) are the critical points of the functional $J$ restricted to the Nehari manifold $\mathcal{M}^{G}$, as defined in (3.3) with $\mu_{1}=\mu_{2}=1 . J$ is an even $\mathcal{C}^{2}$-functional, which is bounded below on $\mathcal{M}^{G}$ by a positive constant. Since $p \in\left(2,2_{N-d}^{*}\right)$ and $\gamma>\max \left\{\gamma_{p}, 0\right\}$ if $\Omega_{0} \neq \emptyset$, Theorem 2.1 implies that $J$ it satisfies $(\mathrm{PS})_{c}$ for every $c \in \mathbb{R}$. Standard variational methods yield a positive minimizer for $J$ on $\mathcal{M}^{G}$, and one can easily adapt [7, Theorem 3.7] to show that $J$ has infinitely many sign-changing $G$-invariant critical points, as claimed.

The next lemma yields Theorem 1.1 for cooperative systems.

Lemma 4.1 Let $w$ be a nontrivial solution to the problem (1.2). Then, there exist $s, t>0$ such that ( $s w, t w)$ is a solution to the system (1.1) if and only if there exists $r>0$ such that

$$
\mu_{1} r^{p-2}+\lambda \alpha r^{\alpha-2}-\lambda \beta r^{\alpha}-\mu_{2}=0 \text { and } \mu_{2}+\lambda \beta r^{\alpha}>0 .
$$

Proof The proof is completely analogous to that of [6, Lemma 4.1].

Proof of Theorem 1.1 for $\lambda>0$. The inequality in (4.1) is automatically satisfied if $\lambda>0$, so (4.1) reduces to assumption $(A)$ and Theorem 1.1 follows from Theorem 1.3. 


\section{References}

1. Badiale, M., Serra, E.: Multiplicity results for the supercritical Hénon equation. Adv. Nonlinear Stud. 4(4), 453-467 (2004)

2. Cao, D., Liu, Z., Peng, S.: Sign-changing bubble tower solutions for the supercritical Hénon-type equations. Ann. Mat. Pura Appl. (4) 197(4), 1227-1246 (2018)

3. Castro, A., Cossio, J., Neuberger, J.M.: A sign-changing solution for a superlinear Dirichlet problem. Rocky Mt. J. Math. 27(4), 1041-1053 (1997)

4. Chen, Z., Zou, W.: Positive least energy solutions and phase separation for coupled Schrödinger equations with critical exponent. Arch. Ration. Mech. Anal. 205(2), 515-551 (2012)

5. Chen, Z., Zou, W.: Positive least energy solutions and phase separation for coupled Schrödinger equations with critical exponent: higher dimensional case. Calc. Var. Partial Differ. Equ. 52(1-2), 423-467 (2015)

6. Clapp, M., Faya, J.: Multiple solutions to a weakly coupled purely critical elliptic system in bounded domains. Discrete Contin. Dyn. Syst. (to appear). Preprint arXiv:1805.10304

7. Clapp, M., Pacella, F.: Multiple solutions to the pure critical exponent problem in domains with a hole of arbitrary size. Math. Z. 259(3), 575-589 (2008)

8. Clapp, M., Pacella, F.: Existence and asymptotic profile of nodal solutions to supercritical problems. Adv. Nonlinear Stud. 17(1), 87-97 (2017)

9. Clapp, M., Pistoia, A.: Existence and phase separation of entire solutions to a pure critical competitive elliptic system. Calc. Var. Partial Differ. Equ. 57, 23 (2018). https://doi.org/10.1007/s00526-017-1283-9

10. Clapp, M., Rizzi, M.: Positive and nodal single-layered solutions to supercritical elliptic problems above the higher critical exponents. Rend. Istit. Mat. Univ. Trieste 49, 53-71 (2017)

11. Conti, M., Terracini, S., Verzini, G.: Nehari's problem and competing species systems. Ann. Inst. Henri Poincaré Anal. Nonlinéaire 19(6), 871-888 (2002)

12. Dávila, J., Faya, J., Mahmoudi, F.: New type of solutions to a slightly subcritical Hénon type problem on general domains. J. Differ. Equ. 263(11), 7221-7249 (2017)

13. dos Santos, E.M., Pacella, F.: Hénon-type equations and concentration on spheres. Indiana Univ. Math. J. 65(1), 273-306 (2016)

14. Gladiali, F., Grossi, M., Neves, S.L.N.: Nonradial solutions for the Hénon equation in $\mathbb{R}^{N}$. Adv. Math. 249, 1-36 (2013)

15. Guo, Y., Li, B., Wei, J.: Entire nonradial solutions for non-cooperative coupled elliptic system with critical exponents in $\mathbb{R}^{3}$. J. Differ. Equ. 256(10), 3463-3495 (2014)

16. Hebey, E., Vaugon, M.: Sobolev spaces in the presence of symmetries. J. Math. Pures Appl. (9) 76(10), 859-881 (1997)

17. Ivanov, S.V., Nazarov, A.I.: On weighted Sobolev embedding theorems for functions with symmetries. (Russian) Algebra i Analiz 18(1), 108-123 (2006) (translation in St. Petersburg Math. J. 18(1), 77-88 (2007))

18. Liu, J., Liu, X., Wang, Z.-Q.: Sign-changing solutions for coupled nonlinear Schrödinger equations with critical growth. J. Differ. Equ. 261(12), 7194-7236 (2016)

19. Nachbin, L.: The Haar Integral. Translated from the Portuguese by Lulu Bechtolsheim. Robert E. Krieger Publishing Co., Huntington (1976)

20. Ni, W.M.: A nonlinear Dirichlet problem on the unit ball and its applications. Indiana Univ. Math. J. 31(6), 801-807 (1982)

21. Peng, S., Peng, Y., Wang, Z.-Q.: On elliptic systems with Sobolev critical growth. Calc. Var. Partial Differ. Equ. 55, 142 (2016)

22. Pistoia, A., Soave, N.: On Coron's problem for weakly coupled elliptic systems. Proc. Lond. Math. Soc. (3) 116(1), 33-67 (2018)

23. Pistoia, A., Tavares, H.: Spiked solutions for Schrödinger systems with Sobolev critical exponent: the cases of competitive and weakly cooperative interactions. J. Fixed Point Theory Appl. 19(1), 407-446 (2017)

24. Pucci, P., Serrin, J.: A general variational identity. Indiana Univ. Math. J. 35(3), 681-703 (1986)

25. Soave, N.: On existence and phase separation of solitary waves for nonlinear Schrödinger systems modelling simultaneous cooperation and competition. Calc. Var. Partial Differ. Equ. 53(3-4), 689-718 (2015)

26. Szulkin, A.: Ljusternik-Schnirelmann theory on $\mathcal{C}^{1}$-manifolds. Ann. Inst. Henri Poincaré Anal. Nonlinéaire 5(2), 119-139 (1988)

Publisher's Note Springer Nature remains neutral with regard to jurisdictional claims in published maps and institutional affiliations. 